\title{
$\beta$-Catenin Expression Correlates With Prognosis in Hepatocellular Carcinoma Patients Treated With Transcatheter Arterial Chemoembolization
}

\author{
XIANGXIAN XU ${ }^{1,2 *}$, DAZHI GAO ${ }^{2 *}$, XIAOLI YUAN ${ }^{3}$, LI LIU $^{2}$, \\ XIANG ZHANG ${ }^{1}$, XIANXIAN LIANG ${ }^{1}$, SUI CHEN ${ }^{1,2}$, MIN AI $^{2}$, BO CHEN $^{2}$, \\ DONGHONG SHI ${ }^{2}$, ZHIJIAN YANG ${ }^{4}$, ROBERT M. HOFFMAN ${ }^{4}$ and JIAN XU ${ }^{1,2}$ \\ ${ }^{1}$ College of Medical Imaging, Xuzhou Medical University, Xuzhou, P.R. China; \\ ${ }^{2}$ Department of Medical Imaging, Jinlin Hospital, Nanjing, P.R. China; \\ ${ }^{3}$ Outpatient Department, Jinlin Hospital, Nanjing, P.R. China; \\ ${ }^{4}$ Anticancer Inc., San Diego, CA, U.S.A.
}

\begin{abstract}
Background/Aim: Transcatheter arterial chemoembolization (TACE) is one of the most widely used palliative therapies for the unresectable hepatocellular carcinoma (HCC). However, a large difference is found in prognosis among patients treated with TACE. The aim of the present study was to investigate the prognostic value of $\beta$-catenin in HCC patients treated with TACE. Materials and Methods: Seventy patients with HCC were included in this study. Expression of $\beta$-catenin was determined by immunohistochemistry in biopsy samples taken before TACE. The patients were treated with TACE and followed-up. Clinicopathological parameters, progression-free survival (PFS) and overall survival (OS) were evaluated to analyze the association of $\beta$-catenin expression with prognosis for HCC patients after TACE. Results: HCC patient biopsies exhibited a significantly higher positive rate of $\beta$-catenin expression $(72.86 \%)$ compared to paracancer normal tissues $(19.44 \%) \quad(p<0.01)$. $\beta$-Catenin expression was closely correlated with tumor differentiation, tumor size, serum $\alpha$ fetoprotein (AFP) level and TACE treatment frequency (all $p<0.05)$. Patients with negative $\beta$-catenin expression had longer PFS and OS after TACE compared to those with positive $\beta$-catenin expression (PFS: 44.2 vs. 14.1 months,
\end{abstract}

This article is freely accessible online.

*These Authors equally contributed to this work.

Correspondence to: Jian Xu, College of Medical Imaging, Xuzhou Medical University, Xuzhou 221004, P.R. China; Department of Medical Imaging, Jinlin Hospital, Nanjing 210002, P.R. China. Tel: +86 13851656307, Fax: +86 2583422444, e-mail:13851656307@163.com

Key Words: $\beta$-Catenin, TACE, hepatocellular carcinoma, prognosis. $p=0.004 ;$ OS: 56.4 vs. 35.9 months, $p<0.001)$. Multivariate Cox regression analysis indicated that $\beta$-catenin expression in HCC patients treated with TACE was an independent prognostic factor for higher PFS and OS. Conclusion: The HCC patients with increased $\beta$-catenin expression have a poor prognosis with lower survival rate.

Hepatocellular carcinoma (HCC) is one of the most common and rapidly progressing cancers related to great fatality worldwide $(1,2)$. Although the patients with $\mathrm{HCC}$ will be treated with surgical resection, liver transplantation, locoregional treatments and chemotherapy, HCC prognosis remains poor because of its high chemo-resistance and recurrence (3-5).

Transcatheter arterial chemoembolization (TACE) is used for the treatment of HCC at an intermediate stage, improving survival of patients (6). A previous report suggested that the combination effect of sorafenib and TACE might contribute to a better prognosis of HCC (7). A significant reduction in tumor size could be achieved by TACE in HCC patients at an advanced stage (8). However, the efficacy of TACE treatment is limited, since distant metastasis and reduced long-term survival are observed in HCC patients after TACE $(9,10)$. Therefore, identification of reliable biomarkers that can be used to predict prognosis of HCC patients would contribute to a more effective clinical management of $\mathrm{HCC}$ treatment with TACE.

Wnt/ $\beta$-catenin pathway has been associated with the development and progression of $\operatorname{HCC}(11,12) . \beta$-Catenin expression levels were reported to be correlated with clinicopathological factors and poor survival rate in patients with $\operatorname{HCC}(13,14)$. However, the relationship between $\beta$-catenin expression and prognosis in hepatocellular carcinoma (HCC) after TACE have not been reported. 
In this study we examined the prognostic value of $\beta$-catenin expression in HCC patients who underwent TACE treatment with the aim to identify a new prognostic approach for TACE treatment and improve the overall survival of HCC patients.

\section{Methods and Materials}

Patients. A total of 70 patients with intermediate or advanced HCC were enrolled in this study. The patients had undergone TACE as initial treatment at Nanjing Jinlin Hospital, between June 2009 and March 2017. All patients were diagnosed with HCC by clinical examination, dynamic computed tomography (CT) or magnetic resonance imaging (MRI), $\alpha$-fetoprotein (AFP) examination, and puncture biopsy pathology prior to TACE treatment. The patients were included according to following criteria: older than 18 years; confirmed by puncture biopsy pathology; no indications for surgery resection or the patients refused the surgical resection; capable of action competence; no history of chemotherapy, radiotherapy or other loco-regional therapy; signed the consent voluntarily; liver function categorized to class $\mathrm{C}$ according to Child-Pugh classification but improved to A after regular treatment. The exclusion criteria were: severely damaged liver function, categorized to class $\mathrm{C}$; poor overall health status and unable to tolerate surgical operation; other medical conditions such as diabetes, lung dysfunction, impaired renal function and severe heart disease; blood circulation and coagulation disorders; serious infection or local active infection; large scale metastasis to multiple organs other than liver and expected survival time less than 3 months. This study was approved by the ethics committee of the Nanjing Jinlin Hospital.

Immunohistochemical analysis. Puncture biopsies were obtained from each patient prior to TACE treatment. Thirty-six normal paracarcinoma tissues were used as normal control. $\beta$-Catenin protein expression was measured by immunohistochemical analysis in formalin-fixed, paraffin-embedded tissue sections. Two serial sections of $4 \mu \mathrm{m}$ were cut from each block and placed onto Super Frost Plus glass-slides (Thermo Fisher Scientific Gerhard Menzel, Braunschweig, Germany). Following deparaffinization in xylene, the slides were rehydrated and washed in Tris-buffered saline. The endogenous peroxidase activity was quenched by $10 \mathrm{~min}$ incubation in a mixture of $3 \%$ hydrogen peroxide solution in $100 \%$ methanol (Sigma, St. Louis, MO, USA). Slides were cleared with Trisbuffered saline and placed at room temperature for $1 \mathrm{~h}$. They were then incubated with monoclonal mouse antibody to human $\beta$-catenin protein (Abcam, Fremont, CA, USA) at 1/100 dilution overnight at $4{ }^{\circ} \mathrm{C}$. Phosphate-buffered saline was used instead of the primary antibody as the negative control. After washing with $\mathrm{pH} 7.4$ phosphate-buffered saline (PBS), the sections were incubated with secondary antibody (Dako REAL EnVision Detection System, Dako, UK) for $30 \mathrm{~min}$ at room temperature. Color development was performed with 3, 3'-diaminobenzidine (DAB). Nuclei were lightly counterstained with hematoxylin. The sections were examined microscopically and interpreted in a blinded fashion by two pathologists. Ten areas were randomly selected and counted at a magnification of $200 \times$. $\beta$-Catenin staining was evaluated semiquantitatively on the basis of the percentage of positively stained cells and classified as follows: 0, no staining or weak staining in $<10 \%$ of cells; $1+$, weak immunostaining in $11 \%-30 \%$ of cells; $2+$, moderate immunostaining in $31 \%-50 \%$ of cells and $3+$, strong immunostaining in $>50 \%$ of cells. The staining pattern was either granular or diffuse. Scores of 0 and $1+$ indicate a negative tumor, while scores of $2+$ and $3+$ were regarded as positive.

TACE treatment. Liver and renal function tests, full blood count, clotting profile, AFP, and viral hepatitis status were checked before TACE. After local anesthesia through 5-10 $\mathrm{ml} 2 \%$ lidocaine (Bayer, Leverkusen, Germany) injection $1 \mathrm{~cm}$ under inguinal ligament, TACE was performed using the following procedures. Using 5 French catheter, superior mesenteric and celiac arteriography was performed initially to assess anatomy, tumor staining, and the tumor-feeding artery. The microcatheter was positioned into or as close as possible to the tumor feeding branch, followed by infusion of an emulsion of 30-50 mg of Pirarubicin Hydrochloride for Injection and 5 to $20 \mathrm{ml}$ of iodized oil (lipiodol; Guerbet, Aulnay sous Bois, France). The amount of chemotherapeutic agents or lipiodol was determined based on the tumor diameter, liver function, peripheral blood count, and vasculature of the tumor. For some patients with large and hypervascular tumors, gelatin sponge particles were used. TACE was repeated at an interval of 1.5 to 3 months, usually until CT scans and AFP levels suggested stabilization of the tumor.

Follow-up. Following TACE, patients were followed up with serum AFP, blood routine, liver function, and enhanced CT or MRI with an interval of 1 to 3 months. Patients were given subsequent TACE treatment according to their clinical examinations. The median follow-up period was 37.2 months (range=3.2-120.1) for survivors. Progress-free survival (PFS) was defined from the date of initial TACE treatment until the date of tumor growth $>20 \%$ or new metastasis. Overall survival (OS) was defined from the date of initial TACE treatment until the date of death or last follow-up.

Statistical analysis. All analyses were performed with IBM SPSS Statistics version 23.0 (Armonk, NY: IBM Corp.). Intergroup comparisons of the clinical variables were analyzed using one-way ANOVA analysis for continuous variables and chi-square test for discrete variables. Survival curves were calculated using the KaplanMeier method and compared by the log-rank test. Furthermore, Cox proportional hazard models and logistic regression models were used for the multivariate analysis concerning survival times and the association of $\beta$-catenin expression status with clinicopathological variables, respectively. All $p$-values were two-sided, with $p<0.05$ considered to indicate statistical significance.

\section{Results}

Increased expression of $\beta$-catenin in HCC tissues. Expression level of $\beta$-catenin was determined with immunohistochemistry in HCC and normal paracancer liver tissues from each patient prior to TACE treatment. As shown in Figure 1 and Table I, HCC tissues exhibited a significantly higher positive rate of $\beta$-catenin expression $(72.86 \%)$ compared to the paracancer tissues $(19.44 \%)(p<0.01)$.

The relationship between $\beta$-catenin expression and the clinicopathologic features of HCC. In order to better understand the relationship between $\beta$-catenin expression and prognosis in HCC patients treated with TACE, the association of $\beta$-catenin 

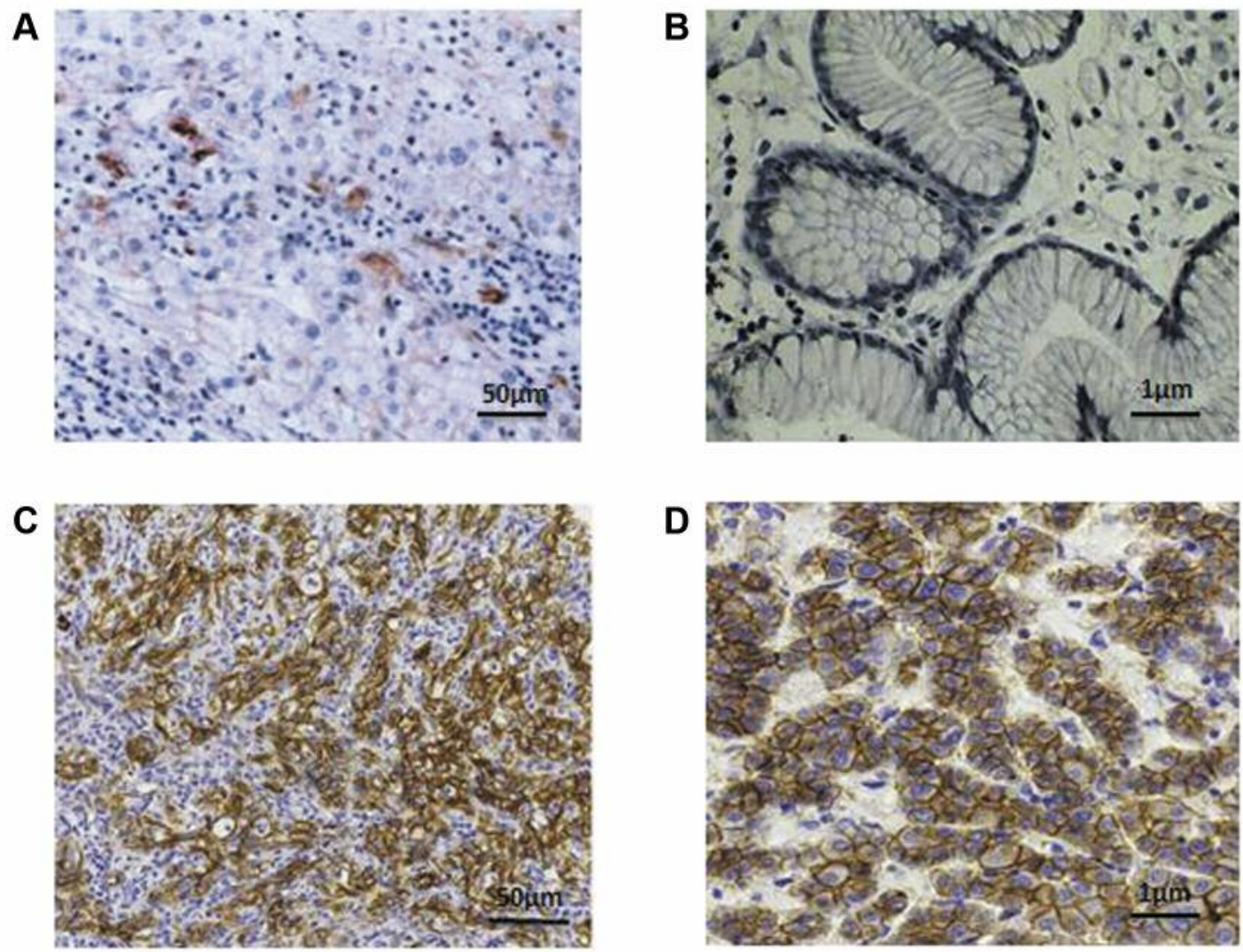

Figure 1. Representative immunohistochemistry staining for $\beta$-catenin expression in hepatocellular carcinoma (HCC) and paracancer tisssues. $\beta$-Catenin expression in paracancer tissue; magnification $200 \times(A) . \beta$-Catenin expression in paracancer tissue; magnification $400 \times(B) . \beta$-Catenin expression in HCC tissue; magnification $200 \times(C)$. D: $\beta$-catenin expression in HCC tissue; magnification $400 \times(D)$.

A

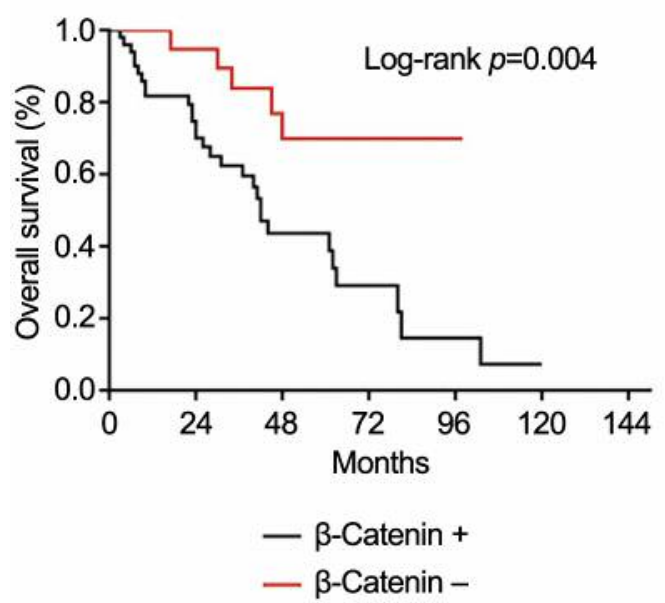

B

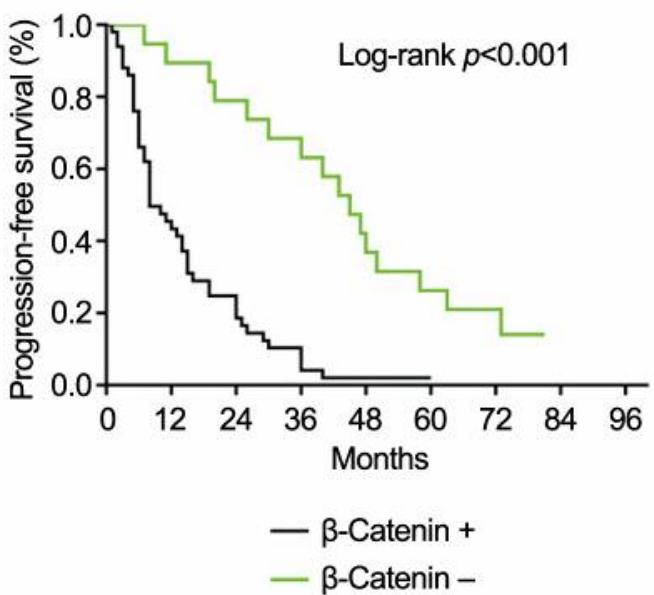

Figure 2. Kaplan-Meier survival analysis of patients treated with TACE $(n=70)$ according to the level of $\beta$-catenin expression, using the median level as cut off. Overall survival of the patients with positive $(n=51)$ and negative $(n=19) \beta$-catenin expression (log-rank, $p=0.004)(A)$. Progressionfree survival of the patients with positive $(n=51)$ and negative $(n=19) \beta$-catenin expression (log-rank, $p<0.001)(B)$. 
Table I. $\beta$-Catenin protein expression in carcinoma and normal paracancer liver tissues.

\begin{tabular}{|c|c|c|c|c|c|}
\hline \multirow[t]{2}{*}{ Tissue type } & \multirow[t]{2}{*}{$\mathrm{N}$} & \multicolumn{2}{|c|}{$\beta$-catenin expression } & \multirow[t]{2}{*}{$\chi^{2}$} & \multirow[t]{2}{*}{$p$-Value } \\
\hline & & $\begin{array}{c}\text { Negative } \\
\mathrm{N}(\%)\end{array}$ & $\begin{array}{c}\text { Positive } \\
\text { N }(\%)\end{array}$ & & \\
\hline Carcinoma & 70 & $19(27.14 \%)$ & $51(72.86 \%)$ & 27.373 & $<0.0001$ \\
\hline Paracancer & 36 & $29(80.55 \%)$ & $7(19.44 \%)$ & & \\
\hline
\end{tabular}

expression with clinicopathological parameters was analyzed. As shown in Table II, the percentage of patients positive for $\beta$-catenin was higher among the patients with poor differentiation than among patients with well/moderate differentiation $(p<0.01)$. The expression of $\beta$-catenin was also positively correlated with tumor size $>5 \mathrm{~cm}$ and AFP $>400 \mathrm{ng} / \mathrm{ml}$ $(p<0.01)$, as well as with TACE treatment frequency $>5$ times $(p<0.01)$. Finally, no relationship was found between $\beta$-catenin expression level and patient gender, age, or Child-Pugh class $(p>0.05)$.

$\beta$-Catenin expression is associated with prognosis in $\mathrm{HCC}$ patients treated with TACE. To investigate whether $\beta$-catenin could be used to predict prognosis after TACE treatment in HCC patients, the Kaplan-Meier method was performed. Survival analysis indicated that patients negative for $\beta$ catenin expression had a longer post-TACE PFS compared to those positive for $\beta$-catenin expression (44.2 vs. 14.1 months, $p=0.004$; Figure $2 \mathrm{~A}$ ). Furthermore, the patients with negative $\beta$-catenin expression had a longer post-TACE OS compared to those with positive $\beta$-catenin expression (56.4 vs. 35.9 months, $p<0.001$; Figure $2 \mathrm{~B}$ ).

Multivariate analysis was performed using the Cox proportional hazards model to analyze the prognostic value of $\beta$-catenin expression; only $\beta$-catenin expression was associated with increased PFS and OS (Table III). AFP level, surgery, pre-treatment tumor size, differentiation, gender, age and TACE treatment frequency did not significantly correlate with either PFS or OS. $\beta$-Catenin expression in HCC patients treated with TACE was an independent prognostic factor for higher PFS [Hazard ratio $(\mathrm{HR})=4.184,95 \%$ confidence interval $(\mathrm{CI})=1.522-11.504, p=0.006]$ and $\mathrm{OS}(\mathrm{HR}=4.479$, $95 \% \mathrm{CI}=2.247-8.925, p=0.000)$.

\section{Discussion}

The present study is the first to demonstrate an association between $\beta$-catenin expression and HCC prognosis after TACE treatment. It was previously reported that inhibition of the $\mathrm{Wnt} / \beta$-catenin signaling pathway improves therapeutic effect of TACE by restraining the migration and invasion, and promoting apoptosis of HCC cells in a rat model of
Table II. Association of $\beta$-catenin protein expression with clinicopathological characteristics in hepatocellular carcinoma patients.

\begin{tabular}{|c|c|c|c|}
\hline \multirow[t]{2}{*}{ Characteristic } & \multicolumn{2}{|c|}{$\beta$-Catenin expression $(n=70)$} & \multirow[b]{2}{*}{$p$-Value } \\
\hline & Negative & Positive & \\
\hline \multicolumn{4}{|l|}{ Gender } \\
\hline Male & 13 & 28 & 0.415 \\
\hline Female & 6 & 23 & \\
\hline \multicolumn{4}{|l|}{ Age } \\
\hline$>50$ & 15 & 35 & 0.554 \\
\hline$<50$ & 4 & 16 & \\
\hline \multicolumn{4}{|l|}{ Child-Pugh class } \\
\hline $\mathrm{A}$ & 16 & 46 & \\
\hline $\mathrm{B}$ & 3 & 5 & 0.674 \\
\hline \multicolumn{4}{|l|}{ Differentiation } \\
\hline Well/moderate & 17 & 7 & \\
\hline Poor & 2 & 44 & 0.000 \\
\hline \multicolumn{4}{|l|}{ Tumor size } \\
\hline$<5 \mathrm{~cm}$ & 15 & 18 & \\
\hline$>5 \mathrm{~cm}$ & 4 & 33 & 0.001 \\
\hline \multicolumn{4}{|l|}{ TACE frequency } \\
\hline$<5$ & 16 & 11 & \\
\hline$>5$ & 3 & 40 & 0.000 \\
\hline \multicolumn{4}{|l|}{ AFP level (ng/ml) } \\
\hline$<400$ & 14 & 19 & \\
\hline$>400$ & 5 & 32 & 0.008 \\
\hline
\end{tabular}

TACE, Transcatheter arterial chemoembolization; AFP, $\alpha$-fetoprotein.

HCC, indicating the $\mathrm{Wnt} / \beta$-catenin signaling pathway might play a crucial role in the prognosis of TACE in patients with HCC (15). However, the clinical investigation for the relationship between $\beta$-catenin expression and prognosis of HCC after TACE has not been explored.

TACE is one of the most widely used palliative therapies for the unresectable $\operatorname{HCC}(16,17)$. TACE exerts an anticancer effect by selectively blocking the arterial blood supply of HCC (18). Two randomized trials showed the survival benefit for the patients with inoperable HCC and the compensated liver function upon receiving TACE treatment $(10,19)$. However, large difference in prognosis was found among patients treated with TACE $(18,20)$. Therefore, finding a reliable molecular biomarker and method to predict prognosis post-TACE treatment becomes imperative as it is critical for the selection of HCC patients and planning of TACE treatment strategy.

Previous studies have reported that serum vascular endothelial growth factor (VEGF) and C-reactive protein (CRP) levels combined with enhanced MRI might serve as markers for efficacy and prognosis evaluation in HCC patients treated with TACE. Low levels of serum VEGF and CRP, small tumor size and low apparent diffusion coeffcient (ADC) value before treatment indicated a better prognosis after TACE (21). 
Table III. Cox multivariate analysis of the association of $\beta$-catenin expression with progression-free survival (PFS) and overall survival (OS) in transcatheter arterial chemoembolization (TACE)-treated hepatocellular carcinoma (HCC) patients.

\begin{tabular}{|c|c|c|c|c|}
\hline \multirow[b]{2}{*}{ Variables } & \multicolumn{2}{|c|}{ PFS } & \multicolumn{2}{|c|}{ OS } \\
\hline & HR $(95 \% \mathrm{CI})$ & $p$-Value & HR $(95 \% \mathrm{CI})$ & $p$-Value \\
\hline \multicolumn{5}{|l|}{$\beta$-catenin } \\
\hline Positive vs. negative & $4.184(1.522-11.504)$ & 0.006 & $4.479(2.247-8.925)$ & 0.000 \\
\hline \multicolumn{5}{|l|}{ AFP } \\
\hline Positive vs. negative & $0.867(0.409-1.836)$ & 0.709 & $1.205(0.698-2.078)$ & 0.504 \\
\hline \multicolumn{5}{|l|}{ Surgery } \\
\hline Yes $v s$. No & $0.841(0.373-1.899)$ & 0.677 & $0.985(0.534-1.787)$ & 0.960 \\
\hline \multicolumn{5}{|c|}{ Pre-treatment tumor diameter $(\mathrm{cm})$} \\
\hline$>5 v s . \leq 5$ & $1.534(0.681-3.453)$ & 0.302 & $1.271(0.732-2.207)$ & 0.394 \\
\hline \multicolumn{5}{|l|}{ Differentiation } \\
\hline Well/moderate $v s$. poor & $0.936(0.417-2.097)$ & 0.872 & $0.640(0.344-1.191)$ & 0.159 \\
\hline \multicolumn{5}{|l|}{ Age (years) } \\
\hline$\geq 60$ vs. $<60$ & $0.534(0.233-1.226)$ & 0.139 & $0.884(0.496-1.576)$ & 0.677 \\
\hline \multicolumn{5}{|l|}{ TACE } \\
\hline$>5 v s . \leq 5$ & $0.619(0.299-1.281)$ & 0.196 & $0.754(0.441-1.288)$ & 0.302 \\
\hline \multicolumn{5}{|l|}{ Gender } \\
\hline Male $v s$. female & $0.522(0.164-1.664)$ & 0.272 & $1.067(0.438-2.598)$ & 0.886 \\
\hline
\end{tabular}

HR, Hazard ratio; CI, confidence interval; AFP, $\alpha$-fetoprotein.

Moreover, it has been demonstrated that glutamine synthetase (GS) could predict adjuvant TACE response in HCC. Patients with a low GS expression who received postoperative adjuvant TACE showed a better OS (22). Regulation of COX-2 expression and epithelial-to-mesenchymal transition by hypoxia-inducible factor- $1 \alpha$ is associated with poor prognosis in HCC patients post TACE surgery (23). Furthermore, a previous report suggested prognostic significance of serum procalcitonin in patients with unresectable HCC treated with TACE (24). However, more biomarkers for better prognosis prediction of TACE still need to be explored. It was reported that TACE might induce significant neoangiogenetic reaction by an increase in VEGF and b-FGF following treatment, which may affect survival of patients (25). The $\mathrm{Wnt} / \beta$-catenin signaling pathway was found to regulate the protein expression of the angiogenic factors, MMP-2, MMP-9, VEGF-A, VEGF$\mathrm{C}$, and bFGF in HCC cells (26). Therefore, we hypothesized that both $\mathrm{Wnt} / \beta$-catenin signaling pathway and TACE may influence HCC outcome, and hence, investigated $\beta$-catenin as a biomarker for HCC prognosis after TACE. The present study is the first to demonstrate that $\beta$-catenin expression is associated with prognosis in HCC patients treated with TACE, suggesting that $\beta$-catenin is worth to be further investigated for its potential as a molecular biomarker for prognosis prediction after TACE treatment.

In conclusion, expression of $\beta$-catenin is increased in HCC tissues and is related to poor differentiation, larger tumor size and higher AFP level. The HCC patients with increased $\beta$-catenin expression have a high TACE treatment frequency and poor prognosis.

\section{Conflicts of Interest}

None of the Authors have any conflict of interest in regard to this study.

\section{Authors' Contributions}

Jian $\mathrm{Xu}, \mathrm{Xiangxian} \mathrm{Xu}$ and Dazhi Gao designed the study and wrote the draft of the manuscript. Xiang Zhang, Xianxian Liang, Sui Chen and Min Ai collected the clinical samples and performed the TACE treatment and follow up. Li Liu, Xiaoli Yuan, Bo Chen and Donghong Shi conducted the immunohistochemical analysis and analyzed the experimental and clinical data. Zhijian Yang and Robert M. Hoffman supervised the overall study and revised the manuscript.

\section{Acknowledgements}

This work was supported by the Chinese National Natural Science Fund (81471632).

\section{References}

1 Jemal A, Bray F, Center MM, Ferlay J, Ward E and Forman D: Global cancer statistics. CA Cancer J Clin 61: 69-90, 2011. PMID: 21296855, doi: 10.3322/caac.20107

2 Ringelhan M, O'Connor T, Protzer U and Heikenwalder M: The direct and indirect roles of $\mathrm{HBV}$ in liver cancer: prospective markers for HCC screening and potential therapeutic targets. J Pathol 235: 355-367, 2015. PMID: 25196558, doi: 10.1002/ path. 4434

3 Desjardins LA: Hepatocellular carcinoma. Clin J Oncol Nurs 6: 107-108, 2002. PMID: 11889672, doi: 10.1188/02.CJON.107-108 
4 Maluccio $\mathrm{M}$ and Covey A: Recent progress in understanding, diagnosing, and treating hepatocellular carcinoma. CA Cancer J Clin 62: 394-399, 2012. PMID: 23070690, doi: 10.3322/ caac. 21161

5 Wang L, Yao M, Dong Z, Zhang Y and Yao D: Circulating specific biomarkers in diagnosis of hepatocellular carcinoma and its metastasis monitoring. Tumour Biol 35: 9-20, 2014. PMID: 24006223, doi: 10.1007/s13277-013-1141-0

6 Lee SW, Lee HL, Han NI, Jang JW, Bae SH, Choi JY and Yoon SK: Early treatment response to transcatheter arterial chemoembolization is associated with time to the development of extrahepatic metastasis and overall survival in intermediatestage hepatocellular carcinoma. Cancer Chemoth Pharm 79: 8188, 2017. PMID: 27913882, doi: 10.1007/s00280-016-3202-8

7 Liu F, Meng Z, Shao G, Wang J, Wang Z, Yang J, Yip CS and He D: Patterns of sorafenib and TACE treatment of unresectable hepatocellular carcinoma in a Chinese population: subgroup analysis of the GIDEON study. Mol Biol Rep 44: 149-158, 2017. PMID: 27981445, doi: 10.1007/s11033-016-4092-x

8 Herber S, Schneider J, Brecher B, Hohler T, Thelen M, Otto G and Pitton MB: TACE: therapy of the HCC before liver transplantation--experiences. Rofo 177: 681-690, 2005. PMID: 15871083, doi: 10.1055/s-2005-858100

9 Qu X-D, Chen C-S, Wang J-H, Yan Z-P, Chen J-M, Gong G-Q, Liu Q-X, Luo J-J, Liu L-X, Liu R and Qian S: The efficacy of TACE combined sorafenib in advanced stages hepatocellullar carcinoma. BMC Cancer 12: 263, 2012. PMID: 22721173, doi: 10.1186/1471-2407-12-263

10 Lo CM, Ngan H, Tso WK, Liu CL, Lam CM, Poon RT, Fan ST and Wong J: Randomized controlled trial of transarterial lipiodol chemoembolization for unresectable hepatocellular carcinoma. Hepatology 35: 1164-1171, 2002. PMID: 11981766, doi: 10.1053/jhep.2002.33156

11 Rogacki K, Kasprzak A and Stępiński A: Alterations of Wnt/ $\beta$ catenin signaling pathway in hepatocellular carcinomas associated with hepatitis C virus. Pol J Pathol 66: 9-21, 2015. PMID: 26017875

12 Gao C, Xiao G and Hu J: Regulation of Wnt/ $\beta$-catenin signaling by posttranslational modifications. Cell Biosci 4: 13-19, 2014. PMID: 24594309, doi: 10.1186/2045-3701-4

13 Gong XY, Ma N, Xu HX, Chen F, Huang XH and Wang Q: Prognostic significance of c-Met, $\beta$-catenin and FAK in patients with hepatocellular carcinoma following surgery. Oncol Lett 15: 3796-3805, 2018. PMID: 29467897, doi: 10.3892/ol.2018.7733

14 Turato C, Vitale A, Fasolato S, Ruvoletto M, Terrin L, Quarta S, Ramirez Morales R, Biasiolo A, Zanus G, Zali N, Tan PS, Hoshida Y, Gatta A, Cillo U and Pontisso P: SERPINB3 is associated with TGF- $\beta 1$ and cytoplasmic $\beta$-catenin expression in hepatocellular carcinomas with poor prognosis. Br J Cancer 110: 2708-2715, 2014. PMID: 24809782, doi: 10.1038/ bjc. 2014.246

15 Wang BM and Li N: Effect of the Wnt// -catenin signaling pathway on apoptosis, migration, and invasion of transplanted hepatocellular carcinoma cells after transcatheter arterial chemoembolization in rats. J Cell Biochem 119: 4050-4060, 2018. PMID: 29232009, doi: 10.1002/jcb.26576

16 Lencioni R: Loco-regional treatment of hepatocellular carcinoma. Hepatology 52: 762-773, 2010. PMID: 20564355, doi: $10.1002 /$ hep. 23725
17 Zhang JB, Chen Y, Zhang B, Xie X, Zhang L, Ge N, Ren Z and Ye SL: Prognostic significance of serum gamma-glutamyl transferase in patients with intermediate hepatocellular carcinoma treated with transcatheter arterial chemoembolization. Eur J Gastroenterol Hepatol 23: 787-793, 2011. PMID: 21730869, doi: 10.1097/MEG.0b013e32834902dd

18 Cheng HY, Wang X, Chen D, Xu AM and Jia YC: The value and limitation of transcatheter arterial chemoembolization in preventing recurrence of resected hepatocellular carcinoma. World J Gastroenterol 11: 3644-3646, 2005. PMID: 15962394

19 Llovet JM, Real MI and Montana X: Arterial embolisation or chemoembolisation versus symptomatic treatment in patients with unresectable hepatocellular carcinoma: a randomised controlled trial. Lancet 359: 1734-1739, 2002. PMID: 12049862 , doi: 10.1016/S0140-6736(02)08649-X

20 Schwartz JD, Schwartz M, Mandeli J and Sung M: Neoadjuvant and adjuvant therapy for resectable hepatocellular carcinoma: review of the randomised clinical trials. Lancet Oncol 3: 593603 2002. PMID: 12372721

$21 \mathrm{Li} \mathrm{Z}$, Xue TQ and Chen XY: Predictive values of serum VEGF and CRP levels combined with contrast enhanced MRI in hepatocellular carcinoma patients after TACE. Am J Cancer Res 6: 2375-2385, 2016. PMID: 27822426

22 Zhang B, Liu K, Zhang J, Dong LW, Jin ZC, Zhang XJ, Xue F and $\mathrm{He} \mathrm{J}$ : Glutamine synthetase predicts adjuvant TACE response in hepatocellular carcinoma. Int J Clin Exp Med 8: 20722-20731, 2015. PMID: 26884995

23 Huang M, Wang L, Chen J, Bai M, Zhou C, Liu S and Lin Q: Regulation of COX-2 expression and epithelial-to-mesenchymal transition by hypoxia-inducible factor- $1 \alpha$ is associated with poor prognosis in hepatocellular carcinoma patients post TACE surgery. Int J Oncol 48: 2144-2154, 2016. PMID: 26984380, doi: 10.3892/ijo.2016.3421

24 Shen H, Zheng S, Chen R, Jin X, Xu X, Jing C, Lin J, Zhang J, Zhang M, Zhang L, Xie X, Guo K, Ren Z, Lin S and Zhang B: Prognostic significance of serum procalcitonin in patients with unresectable hepatocellular carcinoma treated with transcatheter arterial chemoembolization: A retrospective analysis of 509 cases. Medicine (Baltimore) 96(28): e7438, 2017. PMID: 28700480, doi: 10.1097/MD.0000000000007438

25 Sergio A, Cristofori C, Cardin R, Pivetta G, Ragazzi R, Baldan A, Girardi L, Cillo U, Burra P, Giacomin A and Farinati F: Transcatheter arterial chemoembolization (TACE) in hepatocellular carcinoma (HCC): the role of angiogenesis and invasiveness. The Am J Gastroenterol 103: 914-921, 2008. PMID: 18177453, doi: 10.1111/j.1572-0241.2007.01712.x

26 Qu B, Liu BR, Du YJ, Chen J, Cheng YQ, Xu W and Wang XH: Wnt/beta-catenin signaling pathway may regulate the expression of angiogenic growth factors in hepatocellular carcinoma. Oncol Lett 7: 1175-1178, 2014. PMID: 24944688, doi: 10.3892/ ol.2014.1828

Received December 23, 2018 Revised January 19, 2019 Accepted January 24, 2019 